\title{
Hacia una nueva cosmovisión epidemiológica
}

\section{Towards a new epidemiological worldview}

Luego de leer el excelente artículo titulado “Mortalidad infantil en la Cuenca Matanza Riachuelo. Comparación con la Ciudad Autónoma de Buenos Aires (CABA), la provincia de Buenos Aires y la Argentina (de 2010 a 2017)", ${ }^{1}$ de la Dra. Juliana Finkelstein y col., me gustaría comentar varios aspectos que surgen de su lectura.

En primer lugar, hay un análisis lineal de lo claramente expuesto en el artículo: la realidad social y sanitaria de la población que habita dicha cuenca, refleja una realidad alarmante y que merece ser destacada. Asimismo, la situación de la población infantil en particular, reclama una mirada superadora y acciones innovadoras para hacer frente a estos problemas.

En segundo lugar, me gustaría comentar una serie de conceptos que surgen entre líneas y que son de gran relevancia por su propio peso, y también por el contexto epidemiológico actual. Si bien todos están relacionados entre sí, vamos a establecer un análisis en 4 niveles: la mortalidad infantil, la determinación de la salud, la polarización social y epidemiológica y por último reflexionar sobre una nueva concepción de la epidemiología.

¿De qué hablamos cuando nos referimos a tasa de mortalidad infantil? La primera respuesta es muy simple, una tasa específica de mortalidad por edad. Pero no podemos dejar de contemplar que este indicador es, probablemente, uno de los mejores espejos donde puede reflejarse la realidad social y sanitaria de una determinada región. Esta tasa, a diferencia de otras, es atravesada por todos los niveles de determinación de la salud: biológico, social y medioambiental. Cualquier acción que intente modificarla mediante acciones puntuales podrá lograr éxitos parciales, pero sin un enfoque global que contemple todos esos niveles de determinación, la tasa de mortalidad Infantil no se reducirá de un modo sensible y sostenido.

En relación con la determinación de la salud, el artículo nos muestra que un conglomerado poblacional, que comparte condiciones ambientales y sociales adversas, evoluciona de un modo más desfavorable que otro con condiciones mejoradas. Esto, que parece obvio, debe comprenderse en un sentido mucho más amplio que el estudio de grupos poblacionales acotados a una unidad geográfica limitada. La riqueza de este artículo también se refleja al demostrar que, posiblemente, tengan más en común los niños de las comunas de la ribera Matanza-Riachuelo de la CABA con sus vecinos de la provincia de Buenos Aires, que con otras comunas de su propia ciudad. Esto nos obliga a comprender los fenómenos más allá de los límites tradicionales que sesgan cualquier análisis. Se observa que la dinámica poblacional y la dinámica territorial funcionan con tiempos muy distintos. ${ }^{2}$ En general cuando hablamos de determinación social, y en especial cuando consideramos la determinación medioambiental de la salud, ${ }^{3}$ debemos ampliar la perspectiva y contemplar regiones que muchas veces incluyen diferentes provincias o aun países.

Las desigualdades sociales, económicas y de exposición medioambiental tienen un impacto directo en la mortalidad infantil y también en las diferentes morbilidades, especialmente las relacionadas con la primera etapa de la transición de la salud. ${ }^{4}$ Esto, en sociedades con gran nivel de desigualdad socioeconómica, repercute en un proceso llamado polarización epidemiológica. ${ }^{5}$ El resultado no solo se establece directamente sobre los indicadores de salud-enfermedad, sino que también crea un entorno bio-psico-social, nutricional, ambiental, etc. tan dispar, que deja huellas pronunciadas, no solo en el crecimiento del niño, sino en su neurodesarrollo y su capacidad de inclusión social. ${ }^{6} \mathrm{El}$ resultado es que los niños que nacen y sobreviven en la pobreza, la inadecuada nutrición, la exposición a agentes químicos o físicos de los desechos urbanos e industriales, y la cobertura inadecuada del sistema de salud, tendrán menos oportunidades de desarrollarse favorablemente que sus pares nacidos, muchas veces, a pocos kilómetros de estos núcleos altamente vulnerables. Sobra decir que la igualdad de oportunidades es inexistente en situaciones como esta, y su consecuencia es la perpetuación de las diferencias a nivel social y sanitario.

Estas circunstancias aquí expuestas, sumadas a la creación de una nueva conciencia epidemiológica social y sanitaria a partir de la pandemia de COVID-19, representan una oportunidad para proponer una nueva forma de comprender la epidemiología. El abuso del reduccionismo al que ha llegado nuestra profesión nos induce a pensar que los especialistas se dedican solo a su especialidad, y por ello los epidemiólogos a la epidemiología, y esto es un 
enfoque cuestionable. Todos debemos tener pensamiento epidemiológico hasta en la más simple de nuestras acciones diarias, porque la ampliación de las fronteras del conocimiento nos está permitiendo establecer, cada vez con más frecuencia, la relación entre nuestros hábitos de vida y la exposición a determinados agentes, con manifestaciones clínicas antes no vinculadas. Un ejemplo extraordinario lo representa la teoría de Barker, ${ }^{7}$ que relaciona factores perinatales con patología de la vida adulta, como también la concepción de DOHaD "(Developmental Origins of Health and Disease), acrónimo inglés, cuya traducción al español significa origen de la salud y la enfermedad en el desarrollo" ${ }^{\prime 8}$ en los cuales vemos que los factores epigenéticos son influenciados por el medioambiente, y dejan su impronta en varias generaciones.

Hay muchos cambios favorecedores en este sentido, pero también hay fronteras que debemos animarnos a trascender. En primer lugar, parece oportuno romper con la dialéctica sanitaria que se debate entre el enfoque federal y el centralista. Así, como puede ser útil la gestión descentralizada de muchos aspectos de la salud, un nuevo pensamiento epidemiológico, que contemple cohortes que trasciendan el corsé geográfico de las divisiones distritales o aun nacionales, nos permitiría contemplar escenarios más realistas. Esta dualidad, en un mundo con la movilidad y las comunicaciones globalizadas, es un lastre para las medidas de tipo epidemiológico que se requieren en nuestra realidad actual. Las medidas no coordinadas entre distritos diferentes disminuyen las probabilidades de éxito ante los nuevos desafíos sanitarios. Esto no quiere decir que se resigne la autonomía, sino que se debe contemplar la salud de un modo global, con acciones colegiadas entre diferentes autoridades para generar respuestas comunes más eficaces. La dinámica poblacional que presenta un mundo globalizado no puede ser enfrentada con conceptos estáticos ni soluciones individuales o locales. En este marco hay un elemento que es estructural: la información.

Hoy contamos con recursos no aprovechados en todo su potencial sanitario: Internet, Big Data, ${ }^{9}$ inteligencia artificial aplicada a la epidemiología, redes integradas de sistemas de salud, ${ }^{10}$ la historia clínica universal, etc. deberían ser temas prioritarios a definir en la agenda para una moderna y eficaz vigilancia epidemiológica, no solo a nivel de la patología infectocontagiosa, sino también del reconocimiento de factores de la vida cotidiana que modifican nuestra salud en forma directa o indirecta. El camino hacia una cosmovisión epidemiológica implica aprender de las experiencias pasadas, adaptarse a las herramientas del presente y construir un futuro que se presenta desafiante, pero que puede augurar imprescindibles mejoras sociales y sanitarias.

Dr. Pablo R. Justich Zabala Hospital La Vega - Murcia - España

http:/ / dx.doi.org/10.5546/aap.2020.300

Texto completo en inglés:

http:/ / dx.doi.org/10.5546/aap.2020.eng.300

Cómo citar: Justich Zabala PR. Hacia una nueva cosmovisión epidemiológica. Arch Argent Pediatr 2020;118(5):300-301.

\section{REFERENCIAS}

1. Finkelstein JZ, Codebó Ramalho Luz MO, Feiock LE, Della Rosa G, García S. Mortalidad infantil en la Cuenca Matanza Riachuelo. Comparación con la Ciudad de Buenos Aires, la provincia de Buenos Aires y la Argentina (de 2010 a 2017). Arch Argent Pediatr. 2020; 118(5):313-19.

2. Moro J. Heterogeneidad y fragmentación del subsector público de salud en el Gran Buenos Aires. En: Ariovich A, Chiara M, Di Virgilio MM, Jiménez C, Moro J. Salud, política y territorio en el Gran Buenos Aires. Los Polvorines: Universidad Nacional de General Sarmiento, 2012.Pág.45.

3. Organización Panamericana de la Salud. Determinantes ambientales y sociales de la salud. Washington, DC: OPS; 2010.

4. Hernández-Girón C, Orozco-Núñez E, Arredondo-López A. Modelos conceptuales y paradigmas en salud pública. Rev Salud Pública. 2012; 14(2):315-24.

5. Jarillo-Soto E, López-Arellano O. Salud Pública: Objeto de Conocimiento, Prácticas y Formación. Rev Salud Pública. 2007; 9(1):140-54.

6. Cafiero P, Nápoli S, Dartiguelongue JB. Fisiología del neurodesarrollo. En: Dartiguelongue JB. Fisiología pediátrica aplicada a la clínica. CABA: Journal; 2020. Págs.1-37.

7. Barrera Reyes R, Fernández Carrocera LA. Programación metabólica fetal. Perinatol Reprod Hum. 2015; 29(3):99-105.

8. Gil SM, Cabana J. Concepto DOHaD. En: Sociedad Argentina de Pediatría. PRONAP. 2019; 3(1):19-20.

9. Manrai AK, Patel CJ, Ioannidis JP. In the Era of Precision Medicine and Big Data, Who is normal? JAMA. 2018; 319(19):1981-2.

10. Organización Panamericana de la Salud. Redes Integradas de Servicios de Salud: Conceptos, Opciones de Política y Hoja de Ruta para su Implementación en las Américas. Washington D.C: OPS; 2010. Serie: La Renovación de la Atención Primaria de Salud en las Américas No. 4. [Acceso: 11 de junio de 2020]. Disponible en: https: / / www.paho.org/uru/index. php?option=com_docman\&view=download\&alias=145redes-integrad as-de-servicios-de-salud-apsn4\&category_slug=publicaciones-sistemas-y-serviciosde-salud\&Itemid=307 